\title{
Developmental model of suicide trajectories
}

\author{
Monique Séguin, Guy Beauchamp, Marie Robert, Mélanie DiMambro and Gustavo Turecki
}

\section{Background}

Most developmental studies on suicide do not take into account individual variations in suicide trajectories.

\section{Aims}

Using a life course approach, this study explores developmental models of suicide trajectories.

\section{Method}

Two hundred and fourteen suicides were assessed with mixed methods. Statistical analysis using combined discretetime survival (DTS) and growth mixture modelling (GMM) generated various trajectories, and path analysis (Mplus) identified exogenous and mediating variables associated with these trajectories.

\section{Results}

Two groups share common risk factors, and independently of these major risk factors, they have different developmental trajectories: the first group experienced a high burden of adversity and died by suicide in their early 20s; and the second group experienced a somewhat moderate or low burden of adversity before they took their own life. Structura equation modelling identified variables specific to the early suicide trajectory: conduct and behavioural difficulties, socia isolation/conflicts mediated by school-related difficulties, the end of a love relationship, and previous suicide attempts.

\section{Conclusions}

Psychosocial adversity between 10 and 20 years of age may warrant key periods of intervention.

\section{Declaration of interest}

None.
Research over the past decades has developed complex conceptual models for the interaction between exogenous factors that influence the susceptibility to suicide behaviours and contextual factors acting as proximal variables, ${ }^{1,2}$ which take into account the potential changes in these interactions over the life course. Some models based on clinical research are the result of an attempt to identify a trajectory leading to suicidal behaviour by integrating a number of significant risk variables. The diathesis-stress model ${ }^{3,4}$ suggests a developmental sequence wherein genes, other biological factors and severe childhood adversities may have an impact on the accumulation of negative life events and the development of specific mental health vulnerability. Hence, biological predisposition may cause some individuals to be more sensitive to the effects of stressful events and more likely to choose suicidal acts as a response to unbearable emotional pain. Other models ${ }^{5,6}$ suggest the same type of developmental trajectory in a younger population, starting with early adversity (distal variables), which leads to the development of mental health problems (proximal variables), especially depression, before suicidal behaviours. A great number of these studies have used a cross-sectional design. But findings that prove strong associations in regression analysis cannot easily translate into causal chains. In addition, this type of static picture fails to capture the interactions among distinct factors, the developmental period in which they emerge, and the mechanism by which psychopathology (e.g. depression) becomes an outcome or a risk factor for subsequent development problems.

Developmental psychopathology is defined as the study of the origins and course of individual patterns of behavioural maladaptation ${ }^{7}$ and is based on two main ideas: (a) multideterminism and (b) interaction. Most developmental studies on suicide do not take into account individual variations in suicide trajectories. Causal associations in aetiological modelling have been difficult to establish owing to the fact that it is often unclear when difficulties that are risk factors started. ${ }^{8}$ The effects of development, life events and biographical factors on the development of pathology are widely recognized, both in theory and in practice. Human events occur in sequences and are often cumulative, which must be taken into account when calculating the risk they represent. ${ }^{9}$ However, there is the question of translating such biographical contexts into meaningful life trajectories. To gain a better understanding of the developmental process of suicide trajectories, it is imperative that we change our perspective from 'variable-oriented' to 'person-oriented' research. ${ }^{10}$

Very few studies have used a life course and narrative perspective to sequence the biographical events occurring during development. Our research group has been mapping the events occurring over the lifetime of people who take their own lives. In previous papers we established the presence of two different trajectories leading to suicide: ${ }^{11,12}$ the first trajectory includes significant early developmental difficulties and a high burden of adversity throughout the life course; the second trajectory includes a lesser burden of adversity but a slow decline throughout the life course. Although the underlying hierarchical model is useful, it assumes an even population distribution within the two trajectories. But clearly, the effects of life events are cumulative and interactive, and modelling individual differences requires that assumptions be made about whether to use a multivariate or a multinomial distribution for trajectory parameters. In this paper we report on our path analysis modelling, which takes into account the interaction between personal, clinical and contextual variables leading to different suicide trajectories, using combined discrete-time survival (DTS) and growth mixture modelling $(\mathrm{GMM}){ }^{13}$

\section{Method}

\section{Participant recruitment}

Through an ongoing partnership with the coroner's office, our research group recruits the family members of suicide victims in the province of Quebec (Canada). The protocol established is as follows: after the family receives an introductory letter from the coroner's office, a research assistant follows up with a telephone call. Approximately $75 \%$ of close relatives referred by the coroner's 
office agreed to participate in the study. The average interview process involves three different interviews of $3 \mathrm{~h}$ each. This successful partnership has enabled us to pursue recruitment over the past decade, and we now have a data bank of life course calendars mapping the developmental trajectories of people who died by suicide.

In this study we report life course data for 214 suicide cases: $85 \%$ were males, and the mean age at death was 37 years. Regarding marital status, $49 \%$ were single, $29 \%$ were married or living with a partner, and $21 \%$ were divorced. At the time of death, $54 \%$ were working, and $68 \%$ had completed high school, $14 \%$ junior college and 10\% university.

\section{Measurements}

\section{Interview to determine post-mortem diagnosis}

Suicides were assessed by psychological autopsy, which is a validated method. ${ }^{14-16}$ For the purposes of this interview, we administered semi-structured questionnaires - the Structured Clinical Interview for DSM-IV for both Axis I and Axis II disorders (SCID-I and SCID-II respectively) $)^{17,18}$ - to an informant who had known the deceased well. This procedure has been previously described by our group. ${ }^{19,20}$ In addition, hospital files were examined to corroborate the information and to determine which mental health services the deceased had used. A case vignette was then drafted and discussed by a panel, who determined the post-mortem diagnosis by consensus. A series of studies in the past decade has established the concordance of DSM-IV ${ }^{21}$ diagnoses generated by informant report with chart diagnoses. ${ }^{15,16,22,23}$ Both methods have been proved to have good to excellent reliability.

\section{Interview to establish the life trajectory}

The interview method using the life trajectory calendar was borrowed from life history calendar research. ${ }^{24-26}$ The questionnaire uses a life calendar to reconstruct the major events of an individual's life as an aid to accurate recall of significant life experiences. The purpose of this exercise is to position the onset of psychiatric disorder and the occurrence of major life events within the life course. The life calendar makes it possible to pinpoint the occurrence of specific events (both positive and negative). The length and severity of the events is recorded, and they are classified in one of the following 12 life spheres:

(a) Place of residence and change of permanent address.

(b) Relationship and events with the family of origin (relationship with parents) and changes within the family (with parents, siblings, etc.).

(c) Relationship and events in the affective sphere (affective life, living as couple).

(d) Relationship and events associated with starting a family, and events that occurred in this family or families (relationship with children, extended family).

(e) Onset of interpersonal difficulties (difficulties associated with mental health, suicide attempts, illness, etc.).

(f) Events associated with social life (presence or absence of social support, friends, colleagues).

(g) Events associated with academic life (path, interruptions, successes, failures, etc.).

(h) Events associated with professional life (unemployment, stress at work, promotions, etc.).

(i) Presence of protective factors. (j) Events of loss (bereavement, separations, etc.).

(k) Events of social adversities (financial, legal, etc.).

(1) Seeking and consulting mental health services (types of treatments, etc.).

Each of the 12 spheres has a number of clearly described variables, and the severity and duration of each variable is indicated on the calendar. For example, the parent-child relationship covers any major events occurring from birth to emerging adulthood, including child abuse such as neglect (lack of basic care such as food, bath, clean clothes and medical attention), physical violence (receiving a spanking or being hit harder than a spanking) or sexual violence (fondling, incomplete or complete forced sexual relations); presence of household violence; presence of tensions and discord with parents (being threatened, humiliated or ridiculed); separation from parents; and health and mental health problems of parents.

\section{Event recall and life trajectory data analysis}

The life history calendar attempts to understand and measure the variations in the occurrence of severe experiences and the onset of mental health problems during the life course. It is constructed on the basis of events recalled by family members. To maximise the accuracy of retrospective reporting, we ask participants to use certain documents that will help them to recall events, such as a personal calendar or photo albums, and we had access to medical and psychosocial reports, which were obtained with the families' written consent.

The issue of reliability of reporting, or recalling, does not affect all events equally. For example, it appears that some severe events can be measured with reasonable accuracy. Studies on recall factors indicate that people tend to remember important or major events but not minor events, which contributes to an underestimation rather than an overestimation of difficulties. ${ }^{27}$ Other authors suggest that the reliability of memories of life events is enhanced when using mixed data capture methods, and especially narrative approaches. ${ }^{28,29}$ Even when efforts are made to maximise recall accuracy, some studies indicate that there are individuals with certain personality traits or attachment styles who will show a cognitive bias in recalling events or reconstructing memories. Finally, although there are limits in the accuracy of reported events, the importance of better understanding the trajectories leading to suicide argues for the inclusion of lifetime experience.

\section{Life trajectory modelling}

This study had three analytic strategies. The first strategy was to combine the events occurring in a life course into a 'summary variable' to measure the total burden of adversity during a specific age period. The value of this global variable, the 'burden of adversity' variable, was determined by a panel of evaluators. When the interviews with participants were completed, clinical case histories (case vignettes) were drafted with the information obtained from all the measurements and submitted to the panel of evaluators, who were independent from the interviewers. The panel was composed of experts (researchers from our team, clinical practitioners, psychiatrists, psychologists) who analysed the life trajectories and gave an overall burden of adversity rating for every 5-year interval. Interviewers sought to accumulate sufficient narrative detail about the life events to allow trained evaluators to assess the key characteristics of the events, following narrative methodology developed by other groups. ${ }^{29,30}$ The evaluators assessed the 'contextual threat' of events by assessing their relative weight within the respondent's developmental 
circumstances. This conceptualisation of contextual threat was borrowed from the morbidity burden or low disease burden approach $^{31-33}$ used to identify the overall morbidity that affects health. It is associated with allostatic load, a concept that links psychosocial stress with the neurobiological and genetic dimensions of mental disorders and suicide. ${ }^{33,34}$ The overall burden assessments ranged from severe (rating of 1 or 2) to moderate ( 3 or 4 ) to low ( 5 or 6 ). Case reference logs were written and used to maintain the same evaluation across all cases. In all cases, the evaluators coded each 5-year period independently before reaching a consensus through discussion. In studies from our group, the intra-pair agreement for each 5-year segment ranged from $76 \%$ to $97 \%$; the lowest agreement was found in the $0-4$ years age group. ${ }^{12}$

The second strategy was to study the burden of adversity over time. Combined DTS and GMM, using the software Mplus Version 7 for Mac, was used to examine the individual variation in burden of adversity within age periods (10-14 years through to $40-44$ years). GMM can identify distinct subpopulations of trajectories a posteriori, and in doing so generates a set of continuous growth factors, which are the intercept, slope and quadratic term. The suicide of individuals is considered as a unique event in time, and in that respect, DTS analysis (generating a proportional odds continuous latent variable) was added to the growth mixture analysis to construct a categorical latent class variable for the heterogeneity of the study sample. The addition of covariates completed the statistical model (Fig. 1). The objective of this method of analysis was to: (a) identify subgroups of people who followed distinct trajectories based on the severity of their burden of adversity; (b) examine the pattern of variation and stability over time for the subgroups in question; (c) estimate the proportion of individuals in each group; and (d) identify covariates that are predictive of the variation in observed burden of adversity between groups. In line with multivariate analysis, burden of adversity scores for the 0-4 and 5-9 age periods were excluded from the model since their distribution did not meet the normality requirement. For each individual, the statistical procedure yielded a probability of being classified in each

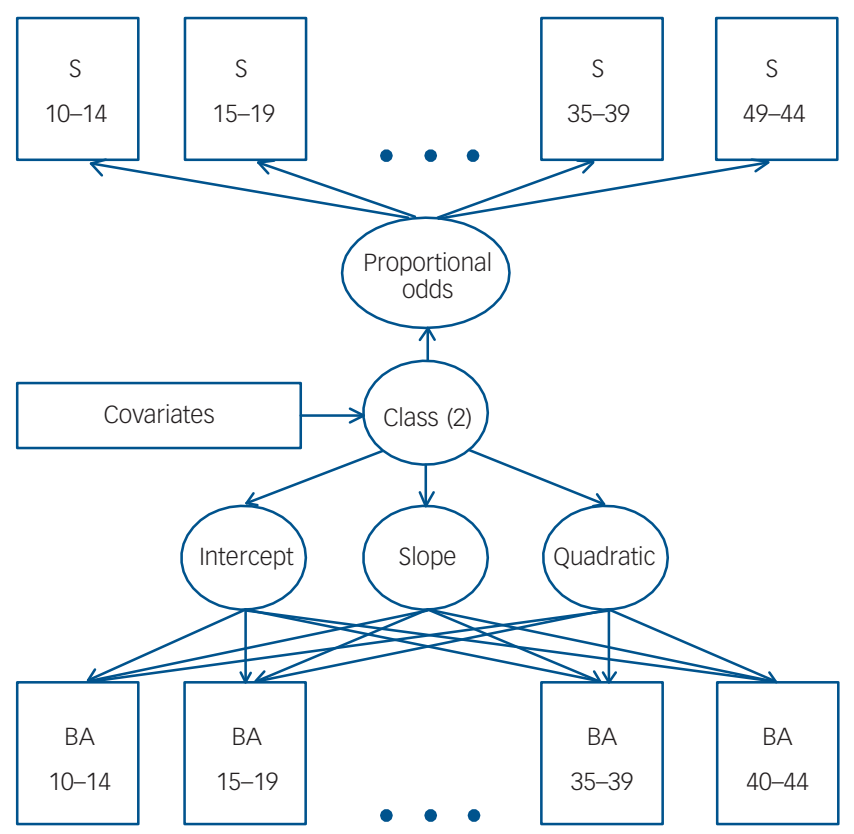

Fig. 1 Combined survival and mixture model explored.

$\mathrm{S}$ indicators correspond to the discrete-time survival (as a dichotomy). BA indicators correspond to the burden of adversity ratings $(1-6)$. Numbers in indicators represent the different age periods. specific group, and group membership was assigned based on the highest probability of classification. Together with the Bayesian information criterion (BIC) and entropy values, these variables provided estimates of model fit and quality of group separation.

The third goal was to develop path models for the variables in each trajectory. Each of the 12 spheres of the life calendar was analysed individually, and the number of events in each 5-year age period was recorded. A series of logistic regression analyses was conducted to determine the significance of each individual risk factor separately in predicting the outcome. Once the significance was established, bivariate relationships between all variables were tested in the form of likelihood ratios (Wald test, zero-order correlations) with the goal of detecting any causal chains. The goal of path analysis is to examine the causal chains between independent variables and find any cause-and-effect relationships between variables. Accordingly, the path model was tested using the following fit indices: comparative fit index (CFI), Tucker-Lewis index (TLI) and root mean square error of approximation (RMSEA). Since all variables were of a categorical nature, path models were fitted with the robust weighted least squares means and variance adjusted estimator. Tetrachoric correlations were established (asymptotic covariance matrix) to produce latent continuous variables. In this framework, we were also able to isolate the direct and indirect effects of all the exogenous variables in the final model. ${ }^{35}$

\section{Results}

\section{Modelling using discrete-time survival mixture analysis}

The longitudinal burden of adversity data were analysed to answer questions related to: (a) establishing the shape of the trajectory; (b) identifying latent classes; and (c) testing predictors for class membership. To select the best-fitted model, we compared the BIC for models with a different number of trajectories. The results shown in Table 1 enabled us to select a model with two trajectories, as the BIC value indicates a good data fit and the entropy value indicates an excellent separation of latent classes. Furthermore, the Lo-Mendell-Rubin likelihood ratio test statistic indicates the greatest improvement for the two-trajectory model. Although the three-trajectory model suggested a slight enhancement, we opted for the two-trajectory model for simplicity and the fact that having two trajectories means each group is represented by a significant number of participants.

Table 2 provides data for the two developmental trajectories found. The average posterior probability assignment for each trajectory is very high ( 0.963 and 0.981 for Trajectories 1 and 2 respectively). Results from a discrete-time survival analysis (Fig. 2) indicate that all members of Trajectory 1 died before the age of 30 . Those in Trajectory 2 had a better survival probability for each age period. The results in Fig. 3 also exhibit

\begin{tabular}{|c|c|c|c|c|}
\hline $\begin{array}{l}\text { Number } \\
\text { of groups }\end{array}$ & $\begin{array}{c}\text { Bayesian information } \\
\text { criterion }\end{array}$ & $\begin{array}{l}\text { Null } \\
\text { model }\end{array}$ & $P^{a}$ & Entropy \\
\hline 1 & 4272.898 & & - & \\
\hline 2 & 3368.692 & 1 & $<0.0001$ & 0.906 \\
\hline 3 & 3364.471 & 2 & $<0.05$ & 0.905 \\
\hline 4 & 3346.880 & 3 & 0.23 & 0.912 \\
\hline
\end{tabular}


the two different trajectories leading to suicide, where the $x$-axis corresponds to the age period and the $y$-axis to the burden of adversity (low burden 5 or 6 , moderate burden 3 or 4 , high burden 1 or 2). Based on this figure, both groups have a similar burden of adversity very early on in life (0-4 years of age, rating near 5), although $10 \%$ of individuals did have a high burden of adversity (value of 1-2). Individuals who followed Trajectory 1 , accounting for $39 \%$ of the sample population, declined rapidly, accumulating a high level of burden of adversity in the following age periods and until early death: $80 \%$ of this subgroup died by suicide during the 20-24 age period (highly significant linear term). However, individuals who followed Trajectory 2, accounting for $61 \%$ of the sample population, were essentially exposed to overall low-to-moderate adversity during their whole lifetime, which varied little (weak linear term). The high significance of the quadratic term (Table 2) is due to the sharp increase in the burden of adversity taking place following the 20-24 age period for this group. Individuals in Trajectory 2 died by suicide at a time when the burden was still somewhat moderate or low.

The sociodemographic data identify very few differences between the two trajectories. Only the 'age at time of death' and 'marital status' variables differed: at time of death individuals in Trajectory 2 were older $(t=18.2$, d.f. $=1, P<0.001)$, and a greater proportion of single participants was observed in Trajectory 1 $\left(\chi^{2}=34.13\right.$, d.f. $\left.=1, P<0.001\right)$. As is usually the case in suicide studies, males were overrepresented, but the gender ratio was identical in both trajectories. Overall, 55\% of participants died on the first suicide attempt, $22 \%$ had had one previous attempt, and $23 \%$ had had more than one previous attempt.

The clinical variables (Table 3 ) suggest that a greater proportion of participants in Trajectory 1 had a first suicide attempt by age 20 (21 v. 10, $\chi^{2}=17.2$, d.f. $=1, P<0.001$ ) and childhood psychopathology (conduct or behavioural difficulties: $\chi^{2}=11.5$, $\mathrm{dF}=1, P<0.001)$. There was no significant difference in either Axis I or Axis II disorders between the trajectories, nor were there significant differences between certain types of life events such as tension, neglect or physical and/or sexual abuse, conduct disorders, parent alcohol misuse and household change.

\section{Modelling using path analysis}

The path analysis model studied is displayed within solid lines in Fig. 4. It clearly delineates the two suicide trajectories and is characterised by adequate fit indices: $\mathrm{CFI}=0.95, \mathrm{TLI}=0.89$, RMSEA $=0.089$ and $R^{2}=0.72$. The mediational effect of school

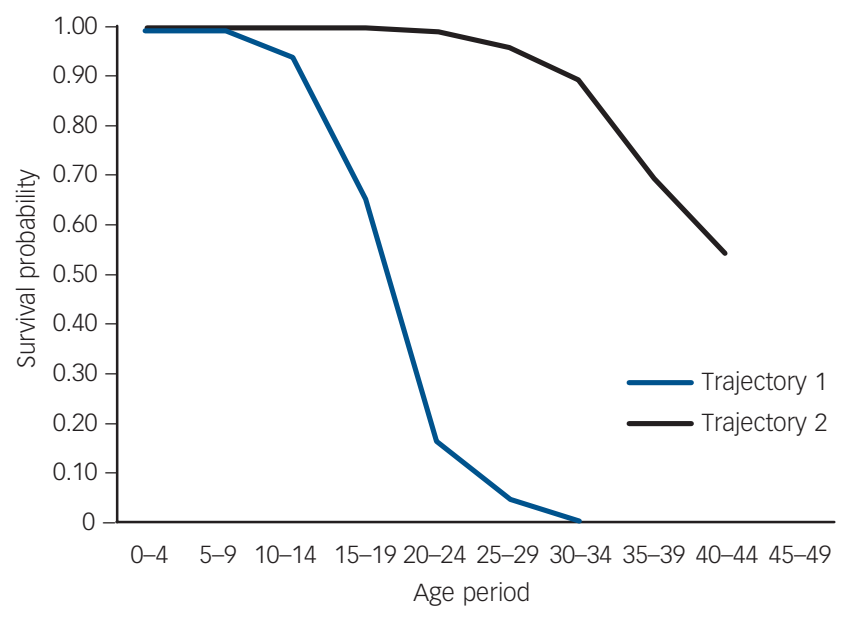

Fig. 2 Survival probability for the two trajectories.

\begin{tabular}{|c|c|c|}
\hline & Trajectory 1 & Trajectory 2 \\
\hline Percentage of sample & 39 & 61 \\
\hline \multicolumn{3}{|l|}{ Parameters, mean (s.e.) } \\
\hline Intercept & $3.519(0.166)^{* * *}$ & $4.506(0.154)^{\star \star *}$ \\
\hline Linear change & $-0.660(0.117)^{\star \star *}$ & $0.145(0.067)^{*}$ \\
\hline Quadratic change & $0.052(0.025)^{*}$ & $-0.053(0.010)^{* * *}$ \\
\hline
\end{tabular}

difficulties on isolation/conflict and conduct/behavioural difficulties with others is relative to Trajectory 1 . The dotted lines include observed common risk factors for suicide. Table 4 lists the bivariate associations between all potential covariates as well as the trajectory group membership in a logistic regression statistical framework (relative to Trajectory 1). The strongest association consisted of the end of a love relationship leading to Trajectory 1 (odds ratio 12.5).

\section{Discussion}

A number of risk factors in the first 10 years of the life course appear to be common to all suicides, irrespective of observed trajectory, and more widely, predictive of a broad range of disorders. For example, close to $40 \%$ of both subgroups (44\% for Trajectory 1 and 36\% for Trajectory 2) were victims of physical and/or sexual abuse during the first 10 years of their lives.

Results combining DTS and GMM suggest two distinct developmental trajectories. The first one was experienced by $40 \%$ of the sample: individuals were plagued with early adversities and seemed to accumulate a great number of developmental difficulties very rapidly, thus creating a greater burden of adversity as time passed. Examples of adversity include parent alcohol misuse, presence of physical or sexual abuse, neglect and tension with parents. These were followed by changes of residence and by the development of psychopathology leading to suicide. All of these risk factors have already been identified in the suicide literature, and the developmental sequence for the emergence of these risk factors follows a clear clinical path to the development of psychopathology, to suicidal ideation, and eventually to suicide. Clearly, not all individuals in this trajectory experienced all of

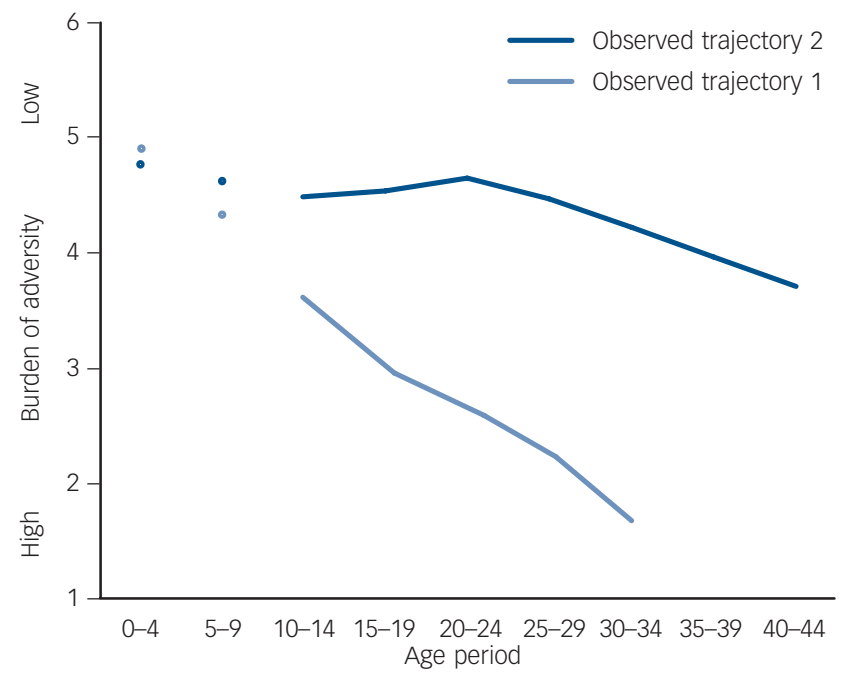

Fig. 3 Trajectory subgroups for two-class survival and growth mixture model. 


\begin{tabular}{|c|c|c|c|c|c|c|c|}
\hline & \multicolumn{2}{|c|}{ Total $(n=214)$} & \multicolumn{2}{|c|}{ Trajectory $1(n=84)$} & \multicolumn{2}{|c|}{ Trajectory $2(n=130)$} & \multirow[b]{2}{*}{$P$} \\
\hline & $n$ & $\%$ & $n$ & $\%$ & $n$ & $\%$ & \\
\hline \multicolumn{8}{|l|}{ Psychopathology } \\
\hline \multicolumn{8}{|l|}{ Past 6 months } \\
\hline Affective disorder & 137 & 64.0 & 57 & 67.9 & 80 & 61.5 & NS \\
\hline Alcohol or substance disorder & 98 & 45.8 & 43 & 51.2 & 55 & 42.3 & NS \\
\hline Anxiety disorder & 29 & 7.4 & 12 & 14.3 & 17 & 13.1 & NS \\
\hline Two or more diagnoses & 124 & 57.9 & 50 & 59.5 & 74 & 56.9 & NS \\
\hline \multicolumn{8}{|l|}{ Lifetime } \\
\hline Affective disorder & 86 & 40.2 & 37 & 44.0 & 49 & 37.7 & NS \\
\hline Alcohol or substance disorder & 113 & 52.8 & 42 & 50.0 & 71 & 54.6 & NS \\
\hline Anxiety disorder & 40 & 18.7 & 19 & 22.6 & 21 & 16.2 & NS \\
\hline Two or more diagnoses & 123 & 57.5 & 52 & 61.9 & 71 & 54.6 & NS \\
\hline Personality disorder & 107 & 50.0 & 43 & 51.2 & 64 & 49.2 & NS \\
\hline Cluster A disorder & 16 & 7.5 & 4 & 4.8 & 12 & 9.2 & NS \\
\hline Cluster B disorder & 48 & 22.4 & 24 & 28.6 & 24 & 18.5 & NS \\
\hline Cluster C disorder & 59 & 27.6 & 22 & 26.2 & 37 & 28.5 & NS \\
\hline \multicolumn{8}{|l|}{ Childhood psychopathology } \\
\hline Conduct disorder only & 25 & 11.7 & 14 & 16.7 & 11 & 8.5 & 0.07 \\
\hline Conduct/behavioural difficulties & 47 & 22.0 & 29 & 34.5 & 18 & 13.8 & $<0.001$ \\
\hline \multicolumn{8}{|l|}{ Clinical risk factors } \\
\hline Abuse (physical/sexual) & 84 & 39.3 & 37 & 44.0 & 47 & 36.2 & NS \\
\hline Parent alcohol misuse & 74 & 34.6 & 28 & 33.3 & 46 & 35.4 & NS \\
\hline Household change & 69 & 32.2 & 34 & 40.5 & 35 & 26.9 & NS \\
\hline Tension & 60 & 28.0 & 28 & 33.3 & 32 & 24.6 & NS \\
\hline Neglect & 50 & 23.4 & 21 & 25.0 & 29 & 22.3 & NS \\
\hline
\end{tabular}

these adversities, but most of them had a rapid increase in the burden of adversity. The goal of this study was to highlight some psychosocial risk factors that have been less resounding, such as social isolation and social conflict before 10 years of age leading to school difficulties coupled with conduct and behavioural difficulties. Two other variables emerged as factors clearly identifying people in Trajectory 1 - the end of a precocious love relationship and a suicide attempt during the teenage years. It sheds light on the sequence of difficulties between early risk variables (physical and sexual aggression, neglect and tension) and variables emerging during the teenage years (conduct and behavioural difficulties, and social and school difficulties) or those emerging during young adulthood (end of a love relationship and suicide attempt). In Trajectory 1, almost every sphere of their lives was affected by adversity, and they died at a very young age, indicating again the importance of and the difficulties in screening for and identifying this type of disruptive, at-risk individual earlier in the developmental process and differentiating between the need for low-intensity and high-intensity intervention.

Individuals in the second trajectory, $60 \%$ of the sample population, were exposed to the same common factors associated with suicide, but had a lower burden of adversity throughout their lives. In some cases, early sexual abuse translated into long-term marital violence, but other spheres of life - academic or professional - were unharmed. This second trajectory was marked by a slower decline over time and was accompanied and compounded by Axis I disorders, such as mood disorders. As events accumulated in the course of their lives and mental health problems created more and more suffering and adversity, these individuals' ability to resist feelings of despair crumbled. In fact these individuals, who do not have a high burden of adversity, may fly under the radar of clinicians, co-workers and, in some cases, close relatives. Perhaps the challenge with this population is to help them access mental health services in a timely manner.

Although in this study we did not measure psychological variables such as temperament, impulsivity, emotion regulation, coping and problem-solving, the psychosocial events observed are an indication of the emergence of mental health difficulties that will be in full bloom during the period of emerging adulthood, and point to the importance of trying to find developmental models that take into account the whole life course. The interaction of genes with early stressors, which may cause changes in the expression of neurotransmitter receptors and neuropeptides - which in turn lowers the thresholds for stress and emotion regulation, decision-making abilities and choice of coping strategies - requires person-oriented research, rather than variable-oriented research. This study raises the question of the existence of common or varying trajectories leading to suicide. The key debate here is how to structure multiple causal influences in the study of suicide trajectories and, unfortunately, very few studies have looked at both predictors and trajectories.

\section{Limitations}

There are a number of limitations to this study. First, the reliability of recalled events was a limitation, even though we made every effort to maximise the accuracy of the retrospective reports. It is important to mention that close family members were interviewed between 4 and 12 months after the death of the individual. For close relatives, grief, but especially grief after suicide, is a period during which they try to understand why the suicide occurred. The interview process echoed the bereavement process and made it easy for close relatives to remember events that occurred over the life course. Nevertheless, the difference between recall with respect to younger individuals and older individuals could also be considered a limitation. The close relatives of those who died at a younger age may have fewer events to remember than close relatives of those who died at an older age. It is also more difficult for the latter group to remember events that happened when the victim was 15 years of age.

Second, the number of variables tested as potential predictors was large, but robust statistical methods were used to ensure statistical validity. Worth noting is the effort made throughout this methodology to maximise the accuracy of the retrospective reports and effectively order within the life course the timeline of events and the onset of mental health problems. Even though 


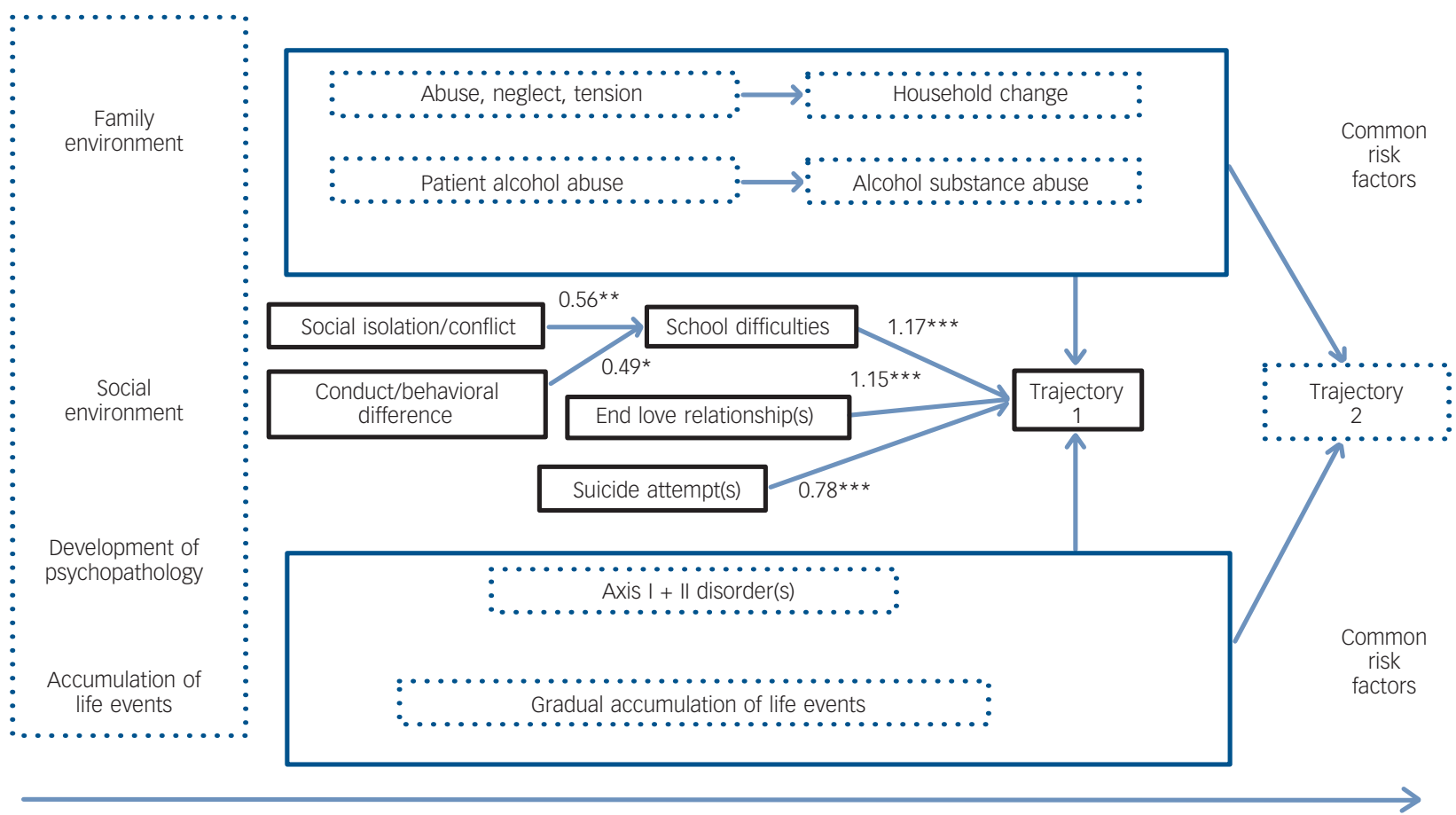

Life course timeline

\section{Fig. 4 Life course model of suicide.}

Path analysis of developmental trajectories with significant covariates included in solid black lines $\left({ }^{*} P<0.05, * \star P<0.01,{ }^{* * *} P<0.001\right)$. Dotted lines outline common risk factors for death by suicide. Values correspond to standardised regression coefficients.

\begin{tabular}{|c|c|c|c|c|c|}
\hline & 1 & 2 & 3 & 4 & 5 \\
\hline \multicolumn{6}{|l|}{ 1. Trajectory (1 relative to 2 ) } \\
\hline 2. Social isolation/conflicts & $2.62 * * *$ & & & & \\
\hline 3. School difficulties & $11.90 * * *$ & $3.05^{* * *}$ & & & \\
\hline 4. End of a love relationship & $12.51 * * *$ & NS & $2.74 * \star$ & & \\
\hline 5. Suicide attempt & $9.43^{* * *}$ & $3.14^{* *}$ & $5.01 * * *$ & $3.24 * *$ & \\
\hline 6. Conduct/behavioural difficulties & $3.24 * * *$ & $7.54^{* *}$ & $2.66^{* *}$ & NS & $5.52^{* \star *}$ \\
\hline
\end{tabular}

this method has its limitations, the narrative methodology is more in harmony with clinical thinking, which demands more than just simple facts and isolated attributes - it requires thinking in terms of conditional and cumulative probabilities and contextual factors.

Third, owing to the small number of females included, our conclusions may not be applicable to both genders, since $85 \%$ of the suicide victims were men. Even though females are equally distributed between the two trajectories, there may be specific events that have a different burden of adversity on the course of men's and women's lives.

\section{Clinical implications}

Social conflict and social isolation, school difficulties, the end of a love relationship, and suicide attempts during teenage years may be symptoms of underlying temperamental difficulties or emerging mental health problems, which rapidly add to the global burden of adversity. The significance of these psychosocial life events argues in favour of school intervention, with early screening and detection of individuals with academic difficulties, conflicts with others, feelings of isolation or rejection in love relationships. These psychosocial problems may be easier to capture in natural settings or within first-line services than identifying emerging mental health issues, especially during youth and adolescence. Early detection and intervention may help alleviate the burden of adversity throughout the life course. More integrated research of life trajectories must be pursued to better understand the key period during which life trajectories may be positively influenced.

\section{Funding}

This study was funded by the Fonds de Recherche du Québec-Santé.

\section{Acknowledgements}

M.S., G.B. and M.R. are with the Département de Psychologie at the Université du Québec en Outaouais. M.S., M.D. and G.T. are with the MCGill Group for Suicide Studies at the Douglas Mental Health University Institute. 
Monique Séguin, PhD, Université du Québec en Outaouais, Department of Psychology, Gatineau, Québec, and McGill Group for Suicide Studies, Douglas Mental Health University Institute, Montreal, Québec; Guy Beauchamp, PhD, Marie Robert PhD, Université du Québec en Outaouais, Department of Psychology, Gatineau, Québec; Mélanie DiMambro, MSc, Gustavo Turecki, MD, PhD, McGill Group for Suicide Studies, Douglas Mental Health University Institute, Montreal, Québec, Canada

Correspondence: Dr Monique Séguin, McGill Group for Suicide Studies, Douglas Mental Health University Institute, Frank B. Common Pavilion, 2nd floor, 6875 LaSalle Blvd, Borough of Verdun, Montreal, Quebec, H4H 1R3 Canada. Email: monique.seguin@uqo.ca

First received 6 Oct 2013, final revision 13 Feb 2014, accepted 20 Feb 2014

\section{References}

1 Beautrais AL. Life course factors associated with suicidal behaviors in young people. Am Behav Sci 2003; 46: 1137-56.

2 Gunnell D, Lewis G. Studying suicide from the life course perspective: implications for prevention. Br J Psychiatry 2005; 187: 206-8.

3 Mann JJ. The neurobiology of suicide. Nat Med 1998; 4: 25-30.

4 Mann JJ. A current perspective of suicide and attempted suicide. Ann Intern Med 2002; 136: 302-11.

5 Bridge JA, Goldstein TR, Brent DA. Adolescent suicide and suicidal behavior. J Child Psychol Psychiatry 2006; 47: 372-94.

6 Wan GWY, Leung PWL. Factors accounting for youth suicide attempt in Hong Kong: a model building. J Adolesc 2010; 33: 575-82.

7 Sroufe LA, Rutter M. The domain of developmental psychopathology. Child Dev 1984; 55: 17-29.

8 Rutter M, Kim-Cohen J, Maughan B. Continuities and discontinuities in psychopathology between childhood and adult life. J Child Psychol Psychiatry 2006; 47: 276-95.

9 Turner RJ, Lloyd DA. Stress burden and the lifetime incidence of psychiatric disorder in young adults: racial and ethnic contrasts. Arch Gen Psychiatry 2004; 61: 481-8.

10 Rutter M, Sroufe LA. Developmental psychopathology: concepts and challenges. Dev Psychopathol 2000; 12: 265-96.

11 Séguin $M$, Renaud J, Lesage $A$, Robert $M$, Turecki G. Youth and young adult suicide: a study of life trajectory. J Psychiatr Res 2011; 45: 863-70.

12 Séguin M, Lesage A, Turecki G, Bouchard M, Chawky N, Tremblay N, et al. Life trajectories and burden of adversity: mapping the developmental profiles of suicide mortality. Psychol Med 2007; 37: 1575-83.

13 Muthen B, Masyn K. Discrete-time survival mixture analysis. J Educ Behav Stat 2005; 30: 27-58

14 Conner KR, Conwell Y, Duberstein PR. The validity of proxy-based data in suicide research: a study of patients 50 years of age and older who attempted suicide. I. Psychiatric diagnoses. Acta Psychiatr Scand 2001; 104 452-7.

15 Kelly TM, Mann JJ. Validity of DSM-III-R diagnosis by psychological autopsy: a comparison with clinician ante-mortem diagnosis. Acta Psychiatr Scand 1996; 94: 337-43.

16 Schneider B, Maurer K, Sargk D, Heiskel H, Weber B, Frolich L, et al. Concordance of DSM-IV Axis I and II diagnoses by personal and informant's interview. Psychiatric Res 2004; 127: 121-36.
17 First MB, Spitzer RL, Gibbon M, Williams JBW. The Structured Clinical Interview for DSM-III-R Personality Disorders (SCID-II). Part I: Description. J Personal Disord 1995; 9: 2-16.

18 Spitzer RL, Williams JB, Gibbon M, First MB. The Structured Clinical Interview for DSM-III-R (SCID). I: History, rationale, and description. Arch Gen Psychiatry 1992; 49: 624-9.

19 Dumais A, Lesage A, Lalovic A, Séguin M, Tousignant M, Chawky N, et al. Is violent method of suicide a behavioral marker of lifetime aggression? Am J Psychiatry 2005; 162: 1375-8.

20 Kim C, Lesage A, Séguin M, Vanier J, Lipp O, Chawky N, et al. Patterns of co-morbidity in male suicide completers. Psychol Med 2003; 33: 1299-1309.

21 American Psychiatric Association. Diagnostic and Statistical Manual of Mental Disorders (4th edn) (DSM-IV). APA, 1994.

22 Brent DA, Perper JA, Moritz G, Allman CJ, Roth C, Schweers J, et al. The validity of diagnoses obtained through the psychological autopsy procedure in adolescent suicide victims: use of family history. Suicide Life Threat Behav 1993; 19: 43-57.

23 Zhang J, Conwell Y, Wieczorek WF, Jiang C, Jia S, Zhou L. Studying Chinese suicide with proxy-based data: reliability and validity of the methodology and instruments in China. J Nerv Ment Dis 2003; 191: 450-7.

24 Caspi A, Moffitt TE, Thornton A, Freedman D, Amell JW, Harrington $H$, et al. The life history calendar: A research and clinical assessment method for collecting retrospective event-history data. Int J Methods Psychiatr Res 1996; 6: 101-14.

25 Moffitt TE, Caspi A, Harington H, Milne BJ. Males on the life-course-persistent and adolescence-limited antisocial pathways: follow-up at age 26 years. Dev Psychopathol 2002; 14: 179-207.

26 Ensel WM, Peek MK, Lin N, Lai G. Stress in the life course: a life history approach. J Aging Health 1996; 8: 389-416.

27 Lin N, Ensel WM, Lai G. Construction and use of the life history calendar: reliability and validity recall data. In Stress and Adversity over the Life Course: Trajectories and Turning Points (eds IH Gotlib, B Wheaton): 249-72. Cambridge University Press, 1997.

28 Brown GW, Andrews B, Bifulco A, Veiel H. Self-esteem and depression 1. Measurement issues and prediction of onset. Soc Psychiatry Psychiatr Epidemiol 1990; 25: 200-9.

29 Dohrenwend BP. Inventorying stressful life events as risk factors for psychopathology: Toward resolution of the problem of intracategory variability. Psychol Bull 2006; 132: 477-95.

30 Brown GW, Harris T. Social Origins of Depression: A Study of Psychiatric Disorder in Women. Free Press, 1978.

31 Powers C, Peckham C. Childhood morbidity and adulthood ill health. J Epidemiol Community Health 1990; 44: 69-74.

32 McGinnis JM, Foege WH. Actual causes of death in the United States JAMA 1993; 270: 2207-12.

33 Forrest $\mathrm{CB}$, Riley AW. Childhood origins of adult health: a basis for life-course health policy. Health Aff (Millwood) 2004; 23: 155-64.

34 McEwen BS. Protective and damaging effects of stress mediators. N Engl J Med 1998; 338: 171-9.

35 Grimm KJ, Ram N. Growth curve modelling from a structural equation modelling perspective. In Handbook of Developmental Research Methods (eds B Laursen, TD Little, NA Card): 411-31. Guilford Press, 2012. 